\title{
Existence of horseshoe maps in current-mode controlled buck-boost dc/dc converters
}

\author{
Dong Dai ${ }^{\mathrm{a}, *}$, Yue Ma ${ }^{\mathrm{b}}$, Chi K. Tse ${ }^{\mathrm{a}, *}$, Xikui Ma ${ }^{\mathrm{c}}$ \\ ${ }^{a}$ Department of Electronic and Information Engineering, Hong Kong Polytechnic University, Hong Kong \\ ${ }^{\mathrm{b}}$ Department of Electrical and Electronic Engineering, University of Tokushima, Tokushima 770-8506, Japan \\ ${ }^{\mathrm{c}}$ School of Electrical Engineering, Xi'anJiaotong University, Xi'an, Shaanxi Province 710049, China
}

Accepted 23 November 2004

\begin{abstract}
In this paper, a buck-boost dc/dc converter under a typical current-mode control is studied. The existence of chaos is proven theoretically in this system. The proof consists of showing that the dynamics of the system is semiconjugate to that of the one-sided shift map, which implies positive entropy of the system and hence chaotic behavior. The essential tool is the horseshoe hypotheses proposed by Kennedy and Yorke, which will be reviewed prior to the discussion of the main finding. Moreover, the existence of chaos is also illustrated in the light of homoclinic intersections of stable and unstable manifolds.
\end{abstract}

(c) 2005 Elsevier Ltd. All rights reserved.

\section{Introduction}

Nonlinear behavior in switching power converters, such as bifurcation and chaos, has attracted much attention from both the engineering and the applied science communities in the past two decades [1-3]. It has been shown that switching power converters can exhibit a variety of complex behavior including saddle-node bifurcation, Hopf bifurcation, period doublings and border collision, as a result of the switching action. Although chaotic behavior has been widely recognized in switching power converter, no rigorous proof of chaos in such systems has been reported. Up to now, only a discussion of the existence of a Smale horseshoe mechanism in a voltage-mode controlled buck converter was reported by Olivar et al. [4]. The main purpose of this paper is to provide a rigorous proof of chaos in a current-mode controlled buck-boost converter. This is realized by proving the existence of a horseshoe map in the describing Poincaré map. The proof is based on the work of Kennedy and Yorke [5], which gives a set of sufficient conditions for a continuous map to exhibit chaotic behavior. Moreover, we illustrate the existence of chaos by observing homoclinic intersections of the stable and unstable manifolds.

\footnotetext{
* Corresponding authors.

E-mail addresses: enddai@eie.polyu.edu.hk (D. Dai), mayue@ee.tokushima-u.ac.jp (Y. Ma), encktse@polyu.edu.hk (C.K. Tse).
} 


\section{The system}

The system studied here is the current-mode controlled buck-boost converter shown in Fig. 1. Bifurcations and chaos in this system have been reported by Dai et al. [6,7]. The state variables for this system are the inductor current, $i$, and the output voltage, $v$. When operating chaotically, this system may operate in discontinuous conduction mode (DCM) for some switching cycles, in addition to the usual continuous conduction mode (CCM) of operation. Thus, to study the chaotic behavior of such a system, we must take into account the possibility of occasional DCM operation in which the inductor current may fall to zero in some switching periods. Suppose we define the Poincaré map as an iterative function that expresses the state variables at the end of a switching period in terms of those at the beginning of the period, i.e.,

$$
x_{n+1}=f\left(x_{n}, \mu\right),
$$

where $x_{n}$ is a vector consisting of the state variables at $t=n T, T$ being the switching period, i.e.,

$$
x_{n}=\left(\begin{array}{c}
i_{n} \\
v_{n}
\end{array}\right)=\left(\begin{array}{c}
i(n T) \\
v(n T)
\end{array}\right) .
$$

Normally the operation can be described as follows. Suppose the period begins with the switch turned on. The inductor current rises linearly, whereas the output voltage decays exponentially. As soon as the inductor current reaches a reference level, $I_{\text {ref }}$, the switch is turned off. Then, the inductor current falls while it delivers current to the load through the conducting diode. The period ends when the switch is turned back on periodically according to the clock signal. Moreover, if the inductor falls to zero before the end of the switching period, the diode will not conduct and the inductor current remains at zero for the rest of the period. Clearly, depending upon the value of $i$ at the beginning of the period and some circuit parameters, the switch may or may not turn off during a switching period. Thus, there are three possible types of operation in any switching period, as illustrated in Fig. 2. The corresponding Poincaré maps can be derived as follows [6,7].

Case a: For the case shown in Fig. 2(a), the inductor current at the beginning of the period is smaller than $I_{\text {ref }}-E T / L$. Thus, if $i_{n} \leqslant I_{\text {ref }}-E T / L$, the Poincaré map is

$$
\left(\begin{array}{c}
i_{n+1} \\
v_{n+1}
\end{array}\right)=\left(\begin{array}{c}
i_{n}+\frac{E T}{L} \\
v_{n} e^{-T / C R}
\end{array}\right) \text {. }
$$

Case b: For the case shown in Fig. 2(b), for which $i_{n}>I_{\text {ref }}-E T / L$ must first be satisfied, the inductor current hits $I_{\text {ref }}$ before the end of the period. In addition, this case corresponds to a CCM operation, i.e., $i_{n+1}>0$. Since the inductor current at the end of the on-time is $I_{\text {ref }}$, the inductor current at any time $t$ during the off-time is

$$
i(t)=e^{-\frac{t t_{\mathrm{ton}}}{2 C R}}\left[I_{\mathrm{ref}} \cos \omega\left(t-t_{\mathrm{on}}\right)-\left(\frac{v_{n} e^{-t_{\mathrm{on}} / C R}}{\omega L}-\frac{I_{\mathrm{ref}}}{2 \omega C R}\right) \sin \omega\left(t-t_{\mathrm{on}}\right)\right],
$$

where $t=0$ is simply taken as the start of the period,

$$
t_{\mathrm{on}}=\frac{L}{E}\left(I_{\mathrm{ref}}-i_{n}\right) \quad \text { and } \quad \omega=\sqrt{\frac{1}{L C}+\frac{1}{4 C^{2} R^{2}}} .
$$

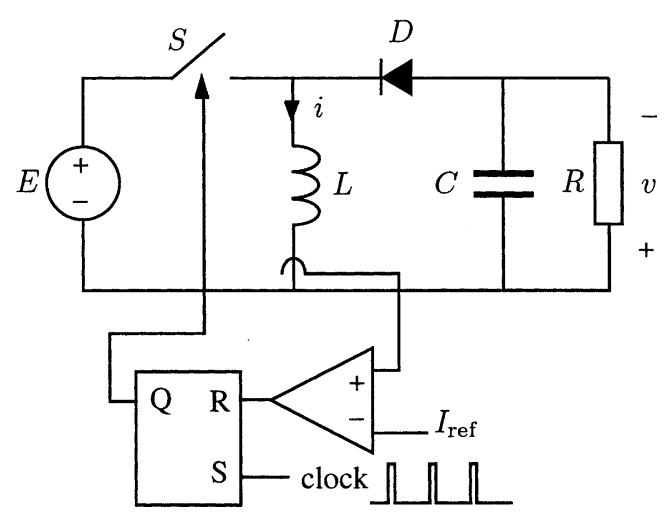

Fig. 1. Current-mode controlled buck-boost converter. 

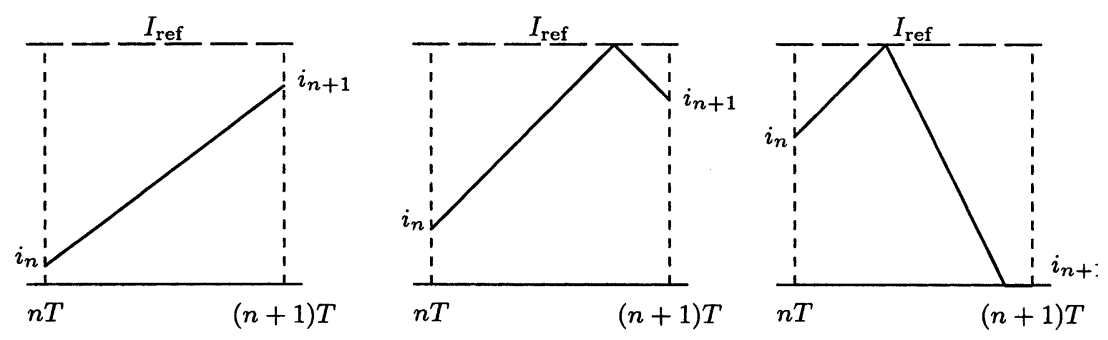

Fig. 2. Inductor current waveforms corresponding to three possible types of operation.

Suppose the inductor current falls to zero at $t=t_{\mathrm{on}}+\xi$. Then, putting $i\left(t_{\mathrm{on}}+\xi\right)=0$ in (4), we have

$$
\xi=\frac{1}{\omega} \arcsin \frac{I_{\mathrm{ref}}}{\sqrt{I_{\mathrm{ref}}^{2}+I_{x}^{2}}}
$$

where, for brevity,

$$
I_{x}=\left(\frac{v_{n} e^{-t_{\mathrm{on}} / C R}}{\omega L}-\frac{I_{\mathrm{ref}}}{2 \omega C R}\right) .
$$

Note that $I_{x}>0$ for most practical circuit conditions. Thus, if $i_{n}>I_{\mathrm{ref}}-E T / L$ and $\xi>T-t_{\mathrm{on}}$, the system maintains a $\mathrm{CCM}$ operation and the Poincaré map is given by

$$
\left(\begin{array}{c}
i_{n+1} \\
v_{n+1}
\end{array}\right)=\left(\begin{array}{c}
\left.e^{-\frac{t_{\mathrm{off}}}{2 C R}\left[I_{\mathrm{ref}} \cos \left(\omega t_{\mathrm{off}}\right)\right.}-I_{x} \sin \left(\omega t_{\mathrm{off}}\right)\right] \\
e^{-\frac{t_{\mathrm{off}}}{2 C R}}\left[v_{n} e^{-t_{\mathrm{on}} / C R} \cos \left(\omega t_{\mathrm{off}}\right)-\left(\frac{v_{n} e^{-\mathrm{on}_{\mathrm{on}} / C R}}{2 \omega C R}-\omega L I_{\mathrm{ref}}\left(1+\frac{1}{4 \omega^{2} C^{2} R^{2}}\right)\right) \sin \left(\omega t_{\mathrm{off}}\right)\right]
\end{array}\right)
$$

where $t_{\mathrm{off}}=T-t_{\mathrm{on}}$.

Case c: Finally, if $i_{n}>I_{\text {ref }}-E T / L$ and $\xi \leqslant T-t_{\text {on }}$, the system will assume a DCM operation, as shown in Fig. 2(c). Clearly, at the instant when $i$ falls to zero, we have

$$
\left(\begin{array}{c}
i\left(t_{\mathrm{on}}+\xi\right) \\
v\left(t_{\mathrm{on}}+\xi\right)
\end{array}\right)=\left(\begin{array}{c}
0 \\
\omega L \sqrt{I_{\mathrm{ref}}^{2}+I_{x}^{2}}
\end{array}\right)
$$

After $i$ has fallen to zero, $v$ simply decays exponentially with a time constant of $C R$. Thus, the Poincaré map for this case is

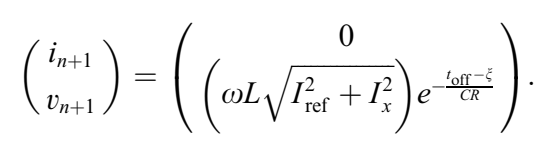

The Poincare map for the system is thus given by (3), (6) and (8), corresponding to an "always on" period, a normal CCM operation and a DCM operation, respectively. As reported in Dai et al. [6,7], this Poincaré map is continuous, but piecewise smooth with two boundaries in the state plane. Intuitively speaking, this map is non-invertible since the injective condition cannot be satisfied, as clearly evidenced from Fig. 2.

To illustrate the behavior of this system, we consider the following set of parameters: $T=200 \mu \mathrm{s}, R=14 \Omega$, $L=0.7 \mathrm{mH}, C=12 \mu \mathrm{F}, E=9 \mathrm{~V}$ and $I_{\text {ref }}=4 \mathrm{~A}$. With this set of parameters, the converter is supposed to operate in CCM with a duty cycle of 0.6544 in the steady state. Thus, as the steady-state duty cycle exceeds 0.5 , the system becomes unstable when it is under current-mode control [8]. A chaotic attractor from the Poincaré map of this system is shown in Fig. 3. 


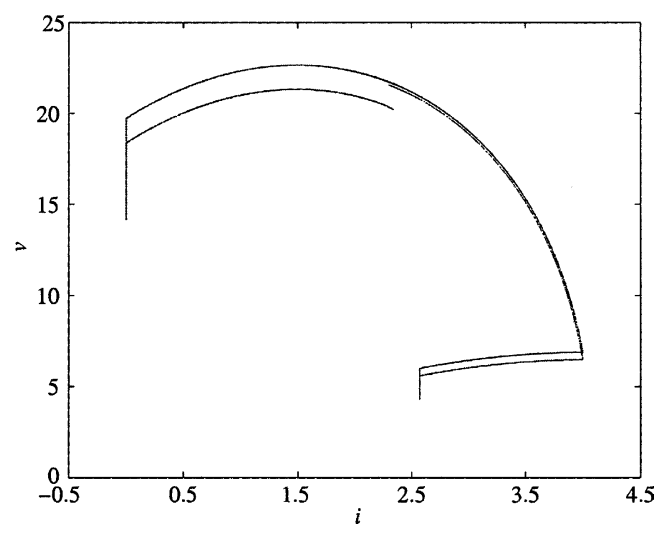

Fig. 3. Chaotic attractor from the current-mode controlled buck-boost converter.

\section{Review of the horseshoe hypotheses}

In this section, we begin with a review of a fundamental result regarding the horseshoe dynamics [9], which is essential to proving the existence of a horseshoe in the current-mode controlled boost converter.

Let $S=\{1,2,3, \ldots, N\}$ be a collection of symbols. Then, a bi-infinite sequence can be constructed with all elements from $S$. Let $\Sigma^{N}$ be the space of all bi-infinite sequences using the symbol set $S$. Any point $s$ in $\Sigma^{N}$ can be written as

$$
s=\left\{\cdots s_{-n} \cdots s_{-1} \cdot s_{0} s_{1} \cdots s_{n} \cdots\right\}
$$

where $s_{i} \in S$ for any $i$. In order to describe limit processes in $\Sigma^{N}$, it is convenient to define a metric on $\Sigma^{N}$. Consider another point $\bar{s}=\left\{\cdots \bar{s}_{-n} \cdots \bar{s}_{-1} \bar{s}_{0} \bar{s}_{1} \cdots \bar{s}_{n} \cdots\right\}$ in $\Sigma^{N}$. The distance between $s$ and $\bar{s}$ is defined as

$$
d(s, \bar{s})=\sum_{i=-\infty}^{\infty} \frac{1}{2|i|} \frac{d_{i}\left(s_{i}, \bar{s}_{i}\right)}{1+d_{i}\left(s_{i}, \bar{s}_{i}\right)},
$$

where

$$
d_{i}\left(s_{i}, \bar{s}_{i}\right)= \begin{cases}1 & \text { if } s_{i} \neq \bar{s}_{i} \\ 0 & \text { if } s_{i}=\bar{s}_{i}\end{cases}
$$

With the distance defined above, the space $\Sigma^{N}$ is a metric space with the metric $d(\cdot, \cdot)$. In the following, we will state some results concerning the structure of $\Sigma^{N}$ without proofs. Readers may refer to Wiggins [9] for detailed proofs.

Proposition 1. The space $\Sigma^{N}$ equipped with the metric defined by Eq. (10) is compact, totally disconnected, and perfect.

The above proposition essentially states the properties that define the structure of the metric space $\Sigma^{N}$. It may be worth noting that compactness, total disconnectedness and perfectness are often taken as the defining properties of a Cantor set, which is used in the characterization of complex structures of invariant sets in a chaotic dynamical system [9].

Proposition 2. Consider the shift map $\sigma: \Sigma^{N} \rightarrow \Sigma^{N}$ which is defined as

$$
\sigma(s)=\left\{\cdots s_{-n} \cdots s_{-1} s_{0} . s_{1} \cdots s_{n} \cdots\right\}
$$

for $s=\left\{\cdots s_{-n} \cdots s_{-1} s_{0} s_{1} \cdots s_{n} \cdots\right\} \in \Sigma^{N}$. The following statements regarding this shift map are true:

1. The metric space $\Sigma^{N}$ is invariant over $\sigma$, i.e., $\sigma\left(\Sigma^{N}\right)=\Sigma^{N}$.

2. $\sigma$ is continuous.

3. $\sigma$ has
(a) a countable infinity of periodic orbits consisting of orbits of all periods;
(b) an uncountable infinity of nonperiodic orbits; and
(c) a dense orbit.

4. $\Sigma^{N}$ is a chaotic, compact invariant set for $\sigma$. 
We remark here that the afore-defined shift map can be equivalently represented by $[\sigma(s)]_{i}=s_{i+1}$.

Next, we will recall some aspects of the topological horseshoe theory proposed by Kennedy and Yorke [5], which are relevant to the main result to be presented in this paper. Essentially, Kennedy and Yorke found a set of sufficient conditions for a continuous map $f$ to exhibit chaos. These conditions are summarized in what has been called the horseshoe hypotheses, which include the following:

1. $X$ is a separable metric space;

2. $Q \subset X$ is locally connected and compact;

3. The map $f: Q \rightarrow X$ is continuous;

4. The sets end en $_{0} \subset Q$ and end $\mathbf{e}_{1} \subset Q$ are disjoint and compact, and each component of $Q$ intersects both end and $_{0}$ end $_{1}$;

5. $Q$ has crossing number $N \geqslant 2$.

The crossing number $N$ in the above hypotheses can be reasoned as follows. A connection $\Gamma$ is a compact connected subset of $Q$ that intersects both sets end end $_{0}$ end $_{1}$. A preconnection $\gamma$ is a compact connected subset of $Q$ for which $\mathrm{f}(\gamma)$ is a connection. Then, the crossing number $N$ is defined as the largest number such that every connection contains at least $N$ mutually disjoint preconnections.

The main result from Kennedy and Yorke can be summarized in the following theorem [5]:

Kennedy-Yorke's Theorem. Consider a map $f$. Suppose the horseshoe hypotheses are satisfied. Then, there is a closed invariant set $\Lambda \subset Q$ for which $f \mid \Lambda$ is semiconjugate to the one-sided shift map on $N$ symbols. ${ }^{1}$

The main consequence of this result is that the entropy of $f$ is not less than that of $\sigma$, which implies that $f$ has positive entropy and is therefore chaotic. Since the theorem provides a weaker set of conditions for proving chaos, it is more readily applied to specific practical systems $[10,11]$.

\section{Horseshoe map in current-mode controlled buck-boost converter}

The Poincaré map for the current-mode controlled buck-boost converter has been defined by (3), (6) and (8). Denote this map by $p$, and consider four iterates of this map, i.e., $p^{4}$. For brevity, we denote $p^{4}$ by $P{ }^{2}$

Consider the rectangle $A B C D$ with the coordinates of four endpoints defined as $A=(2.75,3), B=(3.7,3)$, $C=(3.7,24)$ and $D=(2.75,24)$. Fig. 4 shows the rectangle ABCD and its image $A^{\prime} B^{\prime} C^{\prime} D^{\prime}$ under $P$. Here, $A^{\prime}=P(A)$, $B^{\prime}=P(B), C^{\prime}=P(C)$ and $D^{\prime}=P(D)$. The image $A^{\prime} B^{\prime} C^{\prime} D^{\prime}$ shown in Fig. 4 looks like a curve but is actually a thin band which is topologically similar to the one illustrated in Fig. 5.

Now, we choose the line $A D$ and $B C$ as the two end subsets required in the horseshoe hypotheses. To ease the construction of the preconnections, we look at $A B C D$ and its image $A^{\prime} B^{\prime} C^{\prime} D^{\prime}$ again. As shown in Fig. 4, the map $P$ can map a line to a curve, or a curve to a line. Let $A^{\prime} B^{\prime} C^{\prime} D^{\prime} \cap A D=L_{1}^{\prime} \cup L_{4}^{\prime}$ and $A^{\prime} B^{\prime} C^{\prime} D^{\prime} \cap B C=L_{2}^{\prime} \cup L_{3}^{\prime}$. The preimages of the lines $L_{1}^{\prime}, L_{2}^{\prime}$, $L_{3}^{\prime}$ and $L_{4}^{\prime}$ can be obtained numerically, i.e., $P\left(L_{1}\right)=L_{1}^{\prime}, P\left(L_{2}\right)=L_{2}^{\prime}, P\left(L_{3}\right)=L_{3}^{\prime}$ and $P\left(L_{4}\right)=L_{4}^{\prime}$, as shown in Fig. 6. It should be noted that the zone encompassed by $L_{1}$ and $L_{2}$ within $A B C D$ (denoted by $\Omega_{1}$ ) is disjoint to that by $L_{3}$ and $L_{4}$ (denoted by $\Omega_{2}$ ). It can also be concluded that any curve across the zone $\Omega_{1}\left(\Omega_{2}\right)$ with two ends located on $L_{1}\left(L_{3}\right)$ and $L_{2}\left(L_{4}\right)$ will be a preconnection. This feature is very useful for constructing the proof of the following theorem, which summarizes our main finding.

Main Theorem. For the map $P$ describing the dynamics of the current-mode controlled buck-boost converter, there exists a closed invariant set $\Lambda \subset Q$, where $Q \subset \mathscr{R}^{2}$ such that $P \mid \Lambda$ is semiconjugate to the one-sided shift map, for some chosen parameters.

Proof. Recall the horseshoe hypotheses. First, $\mathscr{R}^{2}$ (which is $\mathrm{X}$ in the horseshoe hypotheses) is separable. Consider $Q=A B C D$ in the afore-described construction. Clearly, $Q \subset X$ is locally connected and compact. The map $P: Q \rightarrow X$ is continuous. Let end ${ }_{0}=A D$ and end ${ }_{1}=B C$. Then, they are disjoint and compact. The crossing number $N$ can be derived as follows. Referring to the afore-presented construction, given any connection $\Gamma$, it will cross both $\Omega_{1}$ and $\Omega_{2}$. Moreover, $\Omega_{1} \cap \Gamma$ and $\Omega_{2} \cap \Gamma$ are two mutually disjoint preconnections as explianed previously. Thus, the crossing number $N \geqslant 2$. Hence, the horseshoe hypotheses are satisfied. From Kennedy-Yorke's theorem, there exists a closed invariant set $\Lambda \subset A B C D$ such that $P \mid \Lambda$ is semiconjugate to the one-sided shift map.

\footnotetext{
${ }^{1}$ A shift map on $N$ symbols is denoted by $\sigma \mid \Sigma^{N}$. If $f \mid \Lambda$ is semiconjugate to $\sigma \mid \Sigma^{N}$, there exists a continuous and onto map $\phi: \Lambda \rightarrow \Sigma^{N}$ such that $\phi \circ f=\sigma \circ \phi$.

${ }^{2}$ The map $P$ can also be regarded as the Poincaré map with stroboscopic sampling period $4 T$.
} 


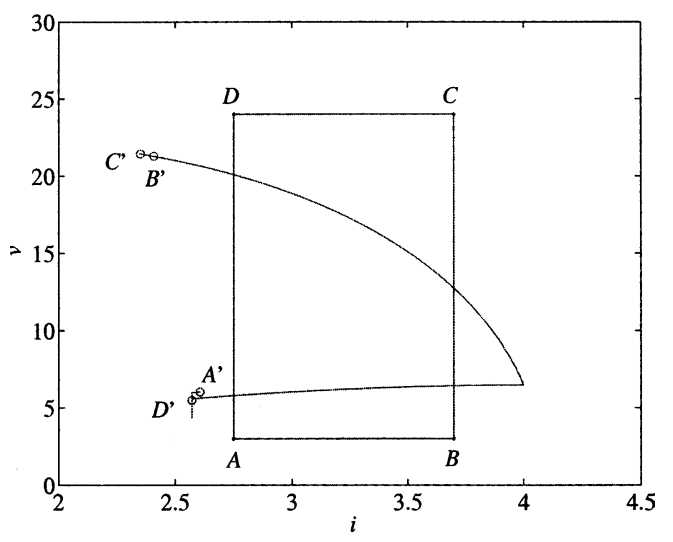

Fig. 4. Rectangle $\mathrm{ABCD}$ and its image $\mathrm{A}^{\prime} \mathrm{B}^{\prime} \mathrm{C}^{\prime} \mathrm{D}^{\prime}$ under $\mathrm{P}$. Images $\mathrm{A}^{\prime}, \mathrm{B}^{\prime}, \mathrm{C}^{\prime}$, and $\mathrm{D}^{\prime}$ are denoted by circles.

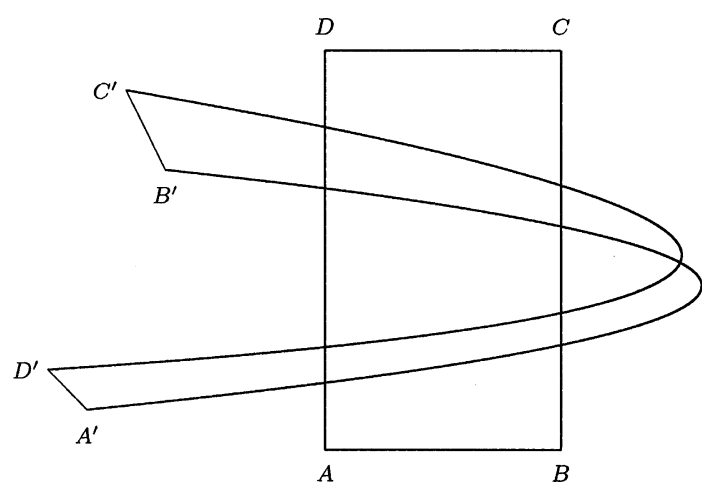

Fig. 5. Illustration of the topological structure of $\mathrm{A}^{\prime} \mathrm{B}^{\prime} \mathrm{C}^{\prime} \mathrm{D}^{\prime}$.

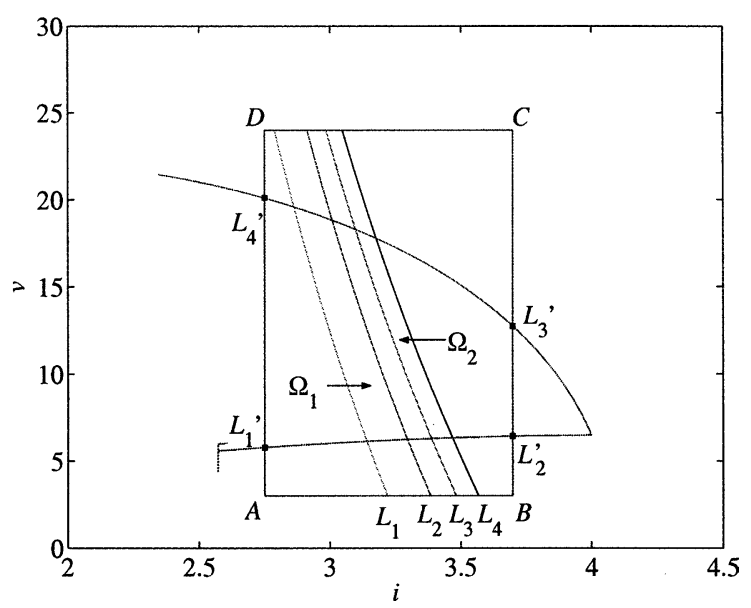

Fig. 6. Intersections of $\mathrm{A}^{\prime} \mathrm{B}^{\prime} \mathrm{C}^{\prime} \mathrm{D}^{\prime}$ with two ends $(\mathrm{AD}$ and $\mathrm{BC})$ and preimages corresponding to these intersections.

\section{Homoclinic intersections}

In this section, we examine the chaotic dynamics of the system from a different viewpoint, which provides indirect evidence of chaos in the system. In studying chaotic dynamics, homoclinic intersections of the stable and unstable 

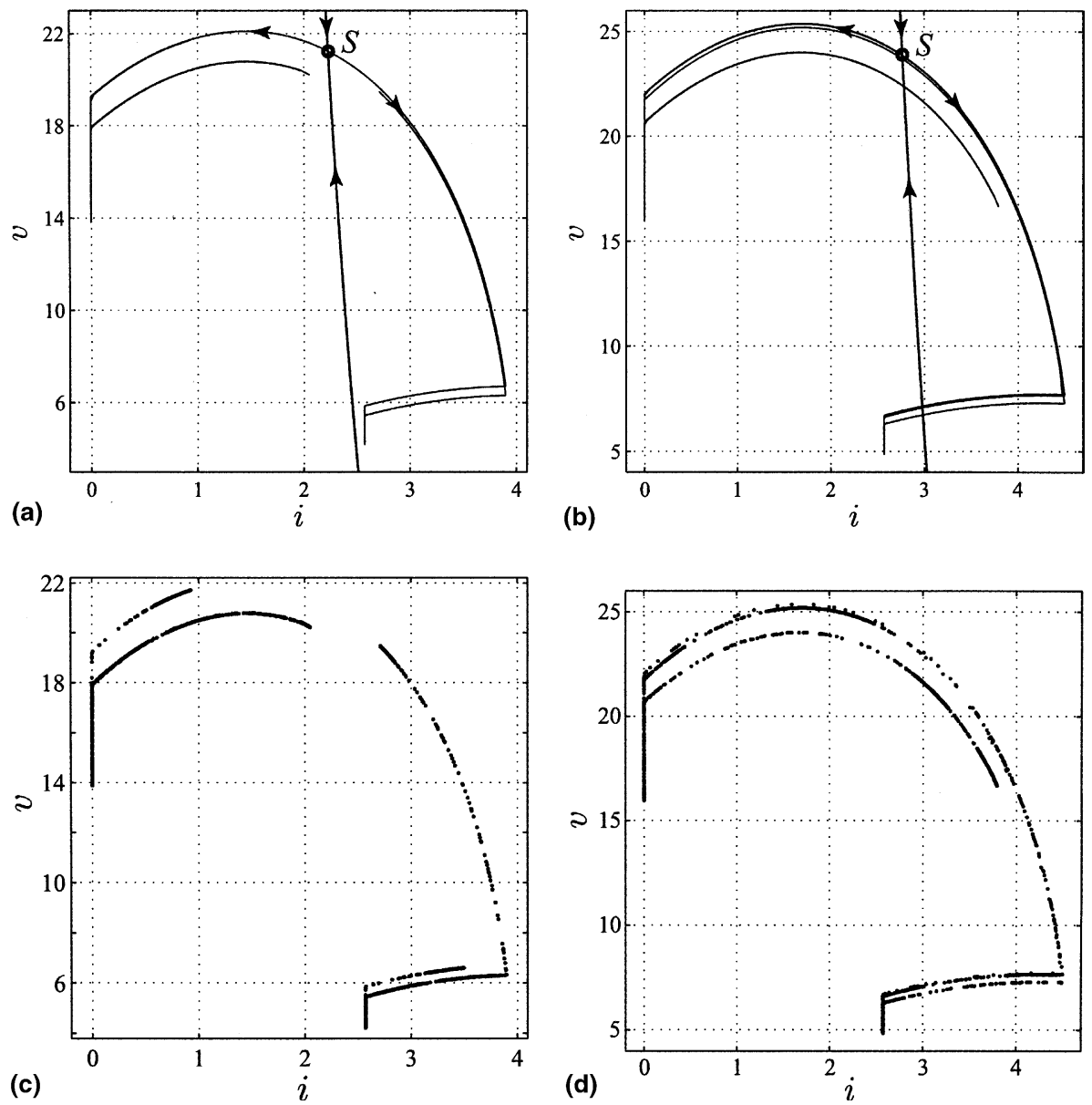

Fig. 7. (a) and (b) - Stable and unstable manifolds of the fixed point $S$. (c) and (d) - Chaotic attractors. $I_{\text {ref }}=3.9$ A for (a) and (c); and $I_{\text {ref }}=4.5 \mathrm{~A}$ for (b) and (d).

manifolds of the same fixed point are often examined [12]. Smale [13] has shown that existence of homoclinic intersection implies horseshoe-type dynamics for some sufficiently large number of iterations of the map. From Fig. 7(a) and (b), we can easily observe homoclinic intersections for the saddle point $S$ from the afore-described Poincaré map $p$, for two values of $I_{\text {ref }}$. Furthermore, as $I_{\text {ref }}$ increases to about $3.997 \mathrm{~A}$, we observe homoclinic tangency, as shown in Fig. 8. Before a homoclinic tangency occurs, the chaotic attractor takes a two-piece localized structure, however, after that, the chaotic attractor becomes a global one, as illustrated in Fig. 7(c) and (d).

From Fig. 7, we see that the chaotic attractors are similar topologically to the unstable manifolds of the saddle point $S$. Thus, we may conjecture that the structure of the chaotic attractor is relevant to that of the invariant manifolds of the saddle point $S$. Finally, we must stress that Smale's result is based on the assumption of an invertible map, and hence cannot be directly applied to our Poincare map $p$ to prove chaos in our system. Nonetheless, the above observed homoclinic intersections clearly illustrate chaos in the Smale's sense although a rigorous proof of chaos based on homoclinic connections could not be pursued due to the non-invertibility of the Poincaré map.

\section{Conclusion}

This paper studies the current-mode controlled buck-boost converter and presents a rigorous proof for the existence of a horseshoe map in the describing Poincare map with the assistance of numerical computation. Our work is based on the result of Kennedy and Yorke [5], which provides a set of sufficient conditions for guaranteeing semiconjugation of a continuous map to the one-sided shift map, which then implies chaotic behavior of the continuous map. Moveover, we 


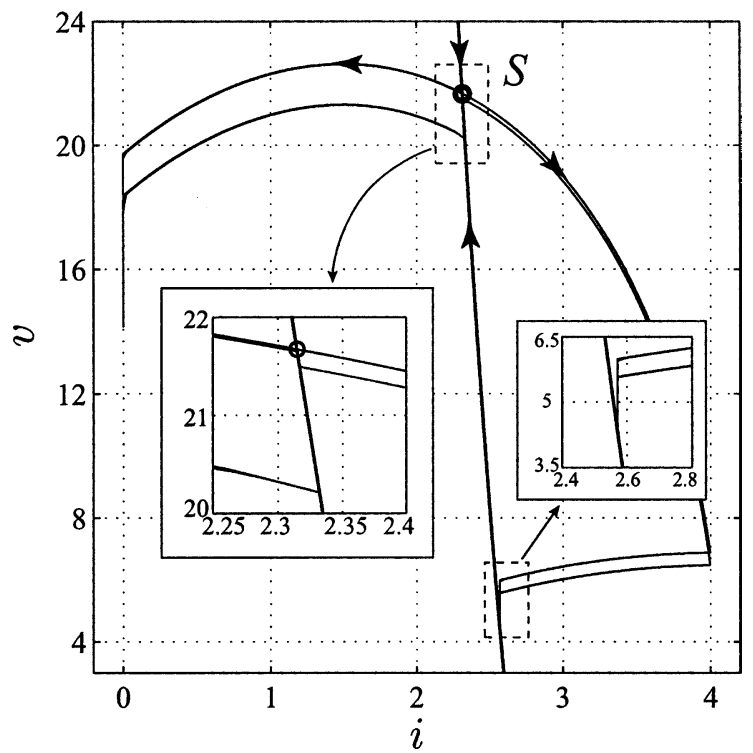

Fig. 8. Invariant manifolds of the fixed point $S$ at homoclinic tangency. Regions around tangency points are enlarged.

also observe the homoclinic intersection of stable and unstable manifolds of the same saddle point, which illustrates the existence of chaos. As very little is known about homoclinic intersection in non-invertible maps, some further investigations are encouraged.

\section{Acknowledgements}

This work is supported by the Hong Kong Research Grant Council under a Competitive Earmarked Research Grant (No. PolyU 5241/03E).

\section{References}

[1] Banerjee S, Verghese G, editors. Nonlinear phenomena in power electronics. IEEE Press; 2000.

[2] Tse CK, Di Bernardo M. Proc IEEE 2002;90:768-81.

[3] Tse CK. Complex behavior of switching power converters. CRC Press; 2003.

[4] Olivar G, Fossas E, Batlle C. Nonlinearity 2000;12:1095-121.

[5] Kennedy J, Yorke JA. Trans Amer Math Soc 2001;353:2513-30.

[6] Dai D, Ma XK, Li XF. Acta Phys Sinica 2003;52:2729-36.

[7] Dai D. Studies on border collision bifurcations in a piecewise-smooth system and the approaches to control and synchronization of chaos. PhD thesis, Xi'an Jiaotong University, China; 2003.

[8] Kislovski AS, Redl R, Sokal NO. Dynamical analysis of switching mode dc/dc converters. Van Nostrand Rein-hold; 1996.

[9] Wiggins S. Introduction to applied nonlinear dynamical systems and chaos. Springer-Verlag; 2003.

[10] Yang XS, Yu YG, Zhang SC. Chaos, Solitons \& Fractals 2003;18:223-7.

[11] Yang XS, Li QD. Int J Bifurcat Chaos 2004;14:1847-52.

[12] Ott E. Chaos in dynamical systems. Cambridge University Press; 2002.

[13] Smale S. Bull Amer Math Soc 1967;73:747-817. 